\title{
Evaluating the diagnostic accuracy of maximal aortic diameter, length and volume for prediction of aortic dissection
}

\author{
Samuel Heuts (D , ${ }^{1,2}$ Bouke P Adriaans, ${ }^{2,3,4}$ Bartosz Rylski, ${ }^{5}$ Casper Mihl, ${ }^{2,3}$ \\ Sebastiaan C A M Bekkers, 2,3,4 Jules R Olsthoorn, ${ }^{1,6}$ Ehsan Natour, ${ }^{1,7}$ Heleen Bouman, ${ }^{8}$ \\ Mikolaj Berezowski, ${ }^{9}$ Kinga Kosiorowska, ${ }^{9}$ Harry J G M Crijns, ${ }^{2,4}$ Jos G Maessen, ${ }^{1,2}$ \\ Joachim Wildberger, ${ }^{2,3}$ Simon Schalla, ${ }^{2,3,4}$ Peyman Sardari Nia ${ }^{1,2}$
}

\begin{abstract}
- Additional material is published online only. To view please visit the journal online (http://dx.doi.org/10.1136/ heartjnl-2019-316251).
\end{abstract}

For numbered affiliations see end of article.

\section{Correspondence to Samuel Heuts, Department of Cardiothoracic Surgery, Maastricht University Medical Centre+, Maastricht, Limburg $6229 H X$, The Netherlands; sam.heuts@mumc.nl}

SH and BPA contributed equally.

Received 15 November 2019 Revised 7 February 2020

Accepted 10 February 2020 Published Online First 8 March 2020

\section{Linked}

- http://dx.doi.org/10.1136/ heartjnl-2020-316617

\section{Check for updates}

(C) Author(s) (or their employer(s)) 2020. No commercial re-use. See rights and permissions. Published by BMJ.

To cite: Heuts $S$, Adriaans BP, Rylski $B$, et al. Heart

2020;106:892-897.

\begin{abstract}
Objective Management of thoracic aortic aneurysms (TAAs) comprises regular diameter follow-up until the indication criterion for prophylactic surgery is reached. However, this approach is unable to predict the majority of acute type $A$ aortic dissections (ATAADs). The current study aims to evaluate the diagnostic accuracy of ascending aortic diameter, length and volume for occurrence of ATAAD.
\end{abstract}

Methods This two-centre observational cohort study retrospectively screened 477 consecutive patients who presented with ATAAD between 2009 and 2018. Of those, 25 (5.2\%) underwent CT angiography (CTA) within 2 years before dissection onset. Aortic diameter, length and volume of these patients ('pre-ATAAD') were compared with those of TAA controls $(n=75)$. Receiver operating curve analysis was performed to evaluate the predictive accuracy of the three different measurements. Results $96 \%$ of patients with pre-ATAAD did not meet the surgical diameter threshold of $55 \mathrm{~mm}$ before dissection onset. Maximal aortic diameters (45 (40-49) $\mathrm{mm}$ vs $46(44-49) \mathrm{mm}, \mathrm{p}=0.075)$ and volume (126 $(95-157) \mathrm{cm}^{3}$ vs $\left.124(102-136) \mathrm{cm}^{3}, p=0.909\right)$ were comparable between patients with pre-ATAAD and TAA controls. Conversely, ascending aortic length $(84 \pm 9 \mathrm{~mm}$ vs $90 \pm 16 \mathrm{~mm}, p=0.031$ ) was significantly larger in patients with pre-ATAAD. All three parameters had an area under the curve of $>0.800$. At the $55 \mathrm{~mm}$ cut-off point, the maximal diameter yielded a positive predictive value (PPV) of $20 \%$. While maintaining same specificity levels, measurements of aortic volume and length showed superior diagnostic accuracy (PPV 55\% and $70 \%$, respectively).

Conclusion Measurements of aortic volume and length have superior diagnostic accuracy compared with the maximal diameter and could improve the timely identification of patients at risk for ATAAD.

\section{INTRODUCTION}

Acute type A aortic dissection (ATAAD) is a medical emergency that carries high morbidity and mortality rates. Only half of patients survive until arrival at the hospital for emergency surgery, and another 20\% of these die perioperatively due to severe disease complications (such as end-organ malperfusion). ${ }^{1-3}$ To date, the maximal aortic diameter is the only recognised anatomic predictor for dissection and rupture. It has been shown that the probability for these to occur increases substantially when crosssectional dimensions exceed $60 \mathrm{~mm} .{ }^{4}$ Since the disruption risk inherent to smaller thoracic aortic aneurysms (TAAs) is low, it does not outweigh the risks of pre-emptive surgery. Therefore, current guidelines recommend referring patients for aneurysm extirpation when the maximal diameter grows beyond $55 \mathrm{~mm} .^{5-7}$ Despite their low risk, the relatively large number of patients with small TAAs cause more than $50 \%$ of dissections to develop in aortas below this size criterion-the so-called 'aortic size paradox'. ${ }^{7-9}$ Since lowering interventional thresholds would not necessarily imply a net mortality benefit (ie, it would expose a large lowrisk population to the considerable risk of surgery), recent studies have debated the clinical value of maximal diameter measurements and focused on the identification of new predictors in an attempt to enhance individualised risk assessment.

Arguably the most important limitation of maximal diameter measurements is the observation that these do not adequately represent the threedimensional process of aortic growth. Elongation and cylindrical deformation (ie, diameter growth proximally or distally from the widest portion of the aneurysm sac) are two scenarios of aortic remodelling that have shown to occur regardless of maximal diameter increase. ${ }^{10} 11$ Therefore, measurements of aortic volume and length could provide additional information over diameters with regard to the detection of aneurysm progression. Whereas the predictive value of aortic volumetry has not yet been investigated, several studies have evaluated the potential role of excessive vessel lengthening in the pathophysiology of dissection. ${ }^{12-14}$ Although all of these studies reported that patients with ATAAD had elongated ascending aortas when compared with healthy controls, these reports mainly included postdissection measurements. This approach is debatable, since it has been shown that the aorta acutely dilates and elongates when it dissects. ${ }^{15}$ In the current retrospective cohort study, we have identified a group of patients who-for various indications-had undergone CT angiography (CTA) within 2 years from ATAAD 
onset. Using this cohort, we were able to assess the geometry of the aorta shortly prior to dissection. By comparing findings with those of TAA controls, this study aimed to evaluate the diagnostic accuracy of ascending aortic diameter, length and volume for the occurrence of ATAAD.

\section{MATERIALS AND METHODS}

\section{Study population}

We retrospectively reviewed the clinical databases of the Maastricht University Medical Center and Heart Centre Freiburg University between January 2009 and December 2018 for patients who were diagnosed with ATAAD and underwent CTA within 2 years before dissection onset. Patients with a history of cardiothoracic surgery, connective tissue disease (such as Marfan's syndrome, Loeys-Dietz or Ehlers-Danlos) or bicuspid aortic valve (BAV) were excluded. The remaining patients were included to form the study population ('pre-ATAAD').

Consecutive patients who underwent CTA at the Mastricht University Medical Center between January 2018 and December 2018 were included in the control groups. Exclusion criteria comprised (suspected) acute aortic syndrome, prior cardiothoracic or aortic surgery, known BAV and presence of any medical condition that might distort aortic shape (such as pulmonary malignancy or pneumothorax). The enrolled patients were subdivided into a group of apparently healthy controls (maximal ascending aortic diameter $<40 \mathrm{~mm}$ ) and a group of patients with TAA (maximal diameter $\geq 40 \mathrm{~mm}$ ). The local ethical committees approved the study protocol and waived the need for informed consent due to the observational character of the study.

\section{Patient and public involvement}

This research was done without patient involvement.

\section{Image acquisition and analysis}

All patients underwent contrast-enhanced CT on a secondgeneration (Maastricht and Freiburg) or third-generation (Maastricht) dual-source scanner (SOMATOM Definition Flash and SOMATOM Force, Siemens Healthineers, Forchheim, Germany, respectively). Patients with pre-ATAAD and TAA typically underwent a dedicated aortic scan protocol that comprised a native CT of the entire aorta, a retrospective ECG-gated helical CT angiography (CTA) of the ascending aorta in caudocranial direction and a non-ECG-triggered high-pitch scan (flash) from the aortic arch to the femoral bifurcation. Scans of the ascending aorta were acquired at slice thickness $0.6 \mathrm{~mm}$ using automated dose modulation (CARE kV, CareDose 4D, Siemens Healthineers). They were reconstructed at an increment of $0.4 \mathrm{~mm}$ using raw-data based iterative reconstruction algorithms (Sinogram Affirmed Iterative Reconstruction (SAFIRE) or Advanced Modelled Iterative Reconstruction (ADMIRE), strength 3, dependent on scanner type). Comprehensive overviews of the various scan parameters per scanner are provided in online supplementary files 1 and 2 . Scans of healthy controls were performed using oncological or vascular workflows, without ECG synchronisation of the respective datasets. Monomeric, non-ionic, lowosmolar iodinated contrast medium $(300 \mathrm{mgI} / \mathrm{mL}$; Iopromide; Bayer Healthcare, Berlin, Germany) was prewarmed to standardised $37^{\circ} \mathrm{C}$ before injection in the antecubital vein using catheter sizes between 18 and 22G. The injection parameters were monitored by a data acquisition system (Certegra Informatics Solution, Bayer Healthcare).

Images were analysed by a single observer who was blinded for patient history (BPA, 5 years of experience in cardiovascular

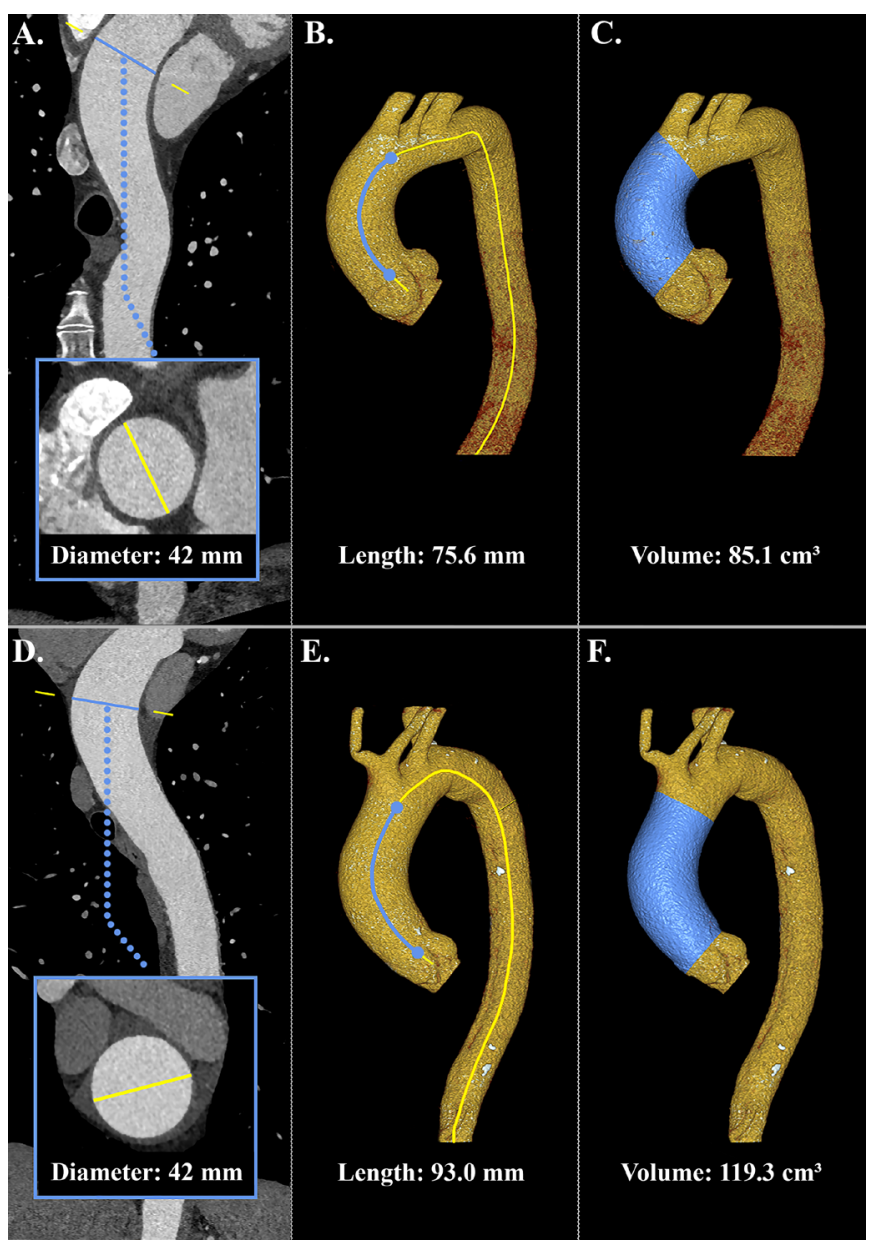

Figure 1 Methodology of the three different aortic measurements in a patient with TAA and a patient with pre-ATAAD.

(A)-(C) demonstrate aortic measurements in a patient with TAA. Panel A depicts a stretched view of the curved planar CT reconstruction with diameter measurement perpendicular to the centreline. The two right panels display a 3D CT model with measurements of ascending aortic length (B) and volume (C). (D)-(F) demonstrate similar measurements in a patient with pre-ATAAD. Despite equal diameters, the patient with pre-ATAAD exhibits a notably longer and larger ascending aorta. ATAAD, acute type $A$ aortic dissection; TAA, thoracic aortic aneurysm; 3D, threedimensional.

imaging), using a commercially available software package ( Syngo.via, Siemens Healthineers). Maximal diameters were measured orthogonal to the vessel centreline using the inner edge to inner edge technique (figure $1 \mathrm{~A}$ and $\mathrm{D}$ ). Measurements of length and volume were performed on a $3 \mathrm{D}$ reconstruction of the thoracic aorta with automatically fitted centreline (figure $1 \mathrm{~B}, \mathrm{C}, \mathrm{E}, \mathrm{F})$. The ascending aorta was defined as the part between the sinotubular junction and the origin of the brachiocephalic trunk. Segment length was measured as the centreline distance between the mentioned anatomic landmarks. The luminal volume was calculated using the adaptive region growing tool provided by the software. Clicking into the vessel initiated a region growing algorithm by segmenting connected voxels of a similar enhancement. Contours were corrected manually in the axial slices when required. Eventually, the volume of the segmentation was calculated automatically by multiplying reconstructed voxel size with the number of voxels included, under consideration of the reconstruction increment. Measurements 
were repeated in a random sample of 20 patients by the same observer and also carried out by a second observer ( $\mathrm{SH}, 3$ years of experience) in order to test intraobserver and interobserver variability.

\section{Statistical analysis}

Statistical analysis of the data was performed using commercially or freely available software (SPSS V.24, IBM, Armonk, New York, USA; or R Statistics, the R Foundation, Vienna, Austria). Continuous data are presented as mean $\pm S D$ (or median and IQR in the presence of skewedness) and were compared using the student $t$-test or Mann-Whitney U-test. Categorical data are expressed as frequencies and percentages and were compared using the $\chi^{2}$ test. $\mathrm{P}<0.05$ was considered statistically significant.

Receiver operating characteristic (ROC) analysis was applied to measure the predictive accuracy of the three measurements, with the calculated area under the curve (AUC) representing the quality of discrimination. The specificity of the current surgical diameter cut-off $(55 \mathrm{~mm})$ was calculated with corresponding sensitivity and used as reference to determine the sensitivities of aortic length and volume at that specificity level. McNemar's test was used to compare sensitivities between groups (DTComPair, R Statistics). ${ }^{16} 17$ In terms of diagnostic accuracy, positive predictive value (PPV) and negative predictive value (NPV) were reported along with $95 \% \mathrm{CI}$.

Finally, intraobserver and interobserver agreement was analysed using intraclass correlation coefficient (ICC) and normalised Bland-Altman analysis.

\section{RESULTS}

The demographic data of the different patient groups are presented in table 1. Between January 2010 and December 2018, a total of 477 patients were diagnosed with ATAAD at the participating institutions. Of those, 25 (5.2\%) had undergone CTA within 2 years before dissection onset. The median time interval between the pre-ATAAD scan and the actual dissection was 124 days (IQR 34-543 days).

In total, 258 healthy controls and 75 patients with TAA were enrolled in the control groups. Scan indications for healthy controls ranged from evaluation of pulmonary nodules $(45.3 \%)$ to suspicion of pulmonary embolism (45.0\%), pneumonia $(5.8 \%)$ and other $(3.9 \%)$. All patients with TAA underwent CT for periodical diameter surveillance.
Table 2 Aortic dimensions of the different patient groups

\begin{tabular}{|c|c|c|c|c|}
\hline & \multirow[b]{2}{*}{$\begin{array}{l}\text { Healthy controls } \\
(n=258)\end{array}$} & \multicolumn{2}{|l|}{ Aortopathy } & \multirow[b]{2}{*}{$P$ value* } \\
\hline & & $\begin{array}{l}\text { TAA controls } \\
(n=75)\end{array}$ & $\begin{array}{l}\text { Pre-ATAAD } \\
(\mathrm{n}=25)\end{array}$ & \\
\hline Diameter (mm) & $33(30-36)$ & $46(44-49)$ & $45(40-49)$ & 0.075 \\
\hline Length (mm) & $66 \pm 9$ & $84 \pm 9$ & $90 \pm 16$ & 0.031 \\
\hline Volume $\left(\mathrm{cm}^{3}\right)$ & $56(45-67)$ & $124(102-136)$ & $126(95-157)$ & 0.909 \\
\hline
\end{tabular}

${ }^{*} P$ values are given for comparison between TAA controls and patients with preATAAD.

ATAAD, acute type $A$ aortic dissections; TAA, thoracic aortic aneurysm.

\section{Aortic dimensions and ROC-curve analysis}

In the pre-ATAAD group, 24 of 25 subjects (96\%) had maximal ascending aortic diameters below the interventional threshold of $55 \mathrm{~mm}$. While diameters and volume were comparable, these patients had significantly longer ascending aortas than those with TAA but no dissection $(90 \pm 16 \mathrm{~mm}$ vs $84 \pm 9 \mathrm{~mm}, \mathrm{p}=0.031)$ (table 2). Scatterplots with correlation coefficients of the various parameters for the different groups are presented in online supplementary file 3 .

All 358 patients were included in the ROC model (dissection $n=25$, control $n=333$ ). Within the entire clinical cohort, all three parameters had an AUC $>0.800$ for prediction of dissection (diameter: 0.810; length 0.842; volume 0.854, all $\mathrm{p}<0.001$; see figure 2A). The current surgical threshold of $55 \mathrm{~mm}$ showed to yield an excellent specificity of $98.8 \%$ (NPV: $93 \%$, CI $90 \%$ to $96 \%$ ). However, the corresponding sensitivity showed to be no more than 4\% (PPV 20\%, CI 0\% to 55\%). At same specificity levels (98.8\%, figure 2B), aortic volume (cut-off: $161 \mathrm{~cm}^{3}$, sensitivity 20\%, $\mathrm{p}=0.045$ ) and length (cut-off: $98 \mathrm{~mm}$, sensitivity $28 \%, p=0.014)$ demonstrated fivefold and sevenfold higher sensitivity for ATAAD detection. Also, PPV of these parameters was markedly higher than that of the maximal diameter (PPV volume: 55\%, CI 23\% to 88\% and PPV length: 70\%, CI 23\% to $88 \%$ ), with similar NPV's (volume 94\%, length 95\%). No statistical significance was reached when comparing the sensitivities of aortic length and volume $(p=0.414)$. Combining the different measurements did not further improve diagnostic performance compared with measurements of aortic length alone.

\section{Intraobserver and interobserver variability}

Intraobserver agreement was excellent for all three parameters (ICC 0.94, 0.97 and 0.99 for aortic diameter, length and volume,

Table 1 Baseline characteristics

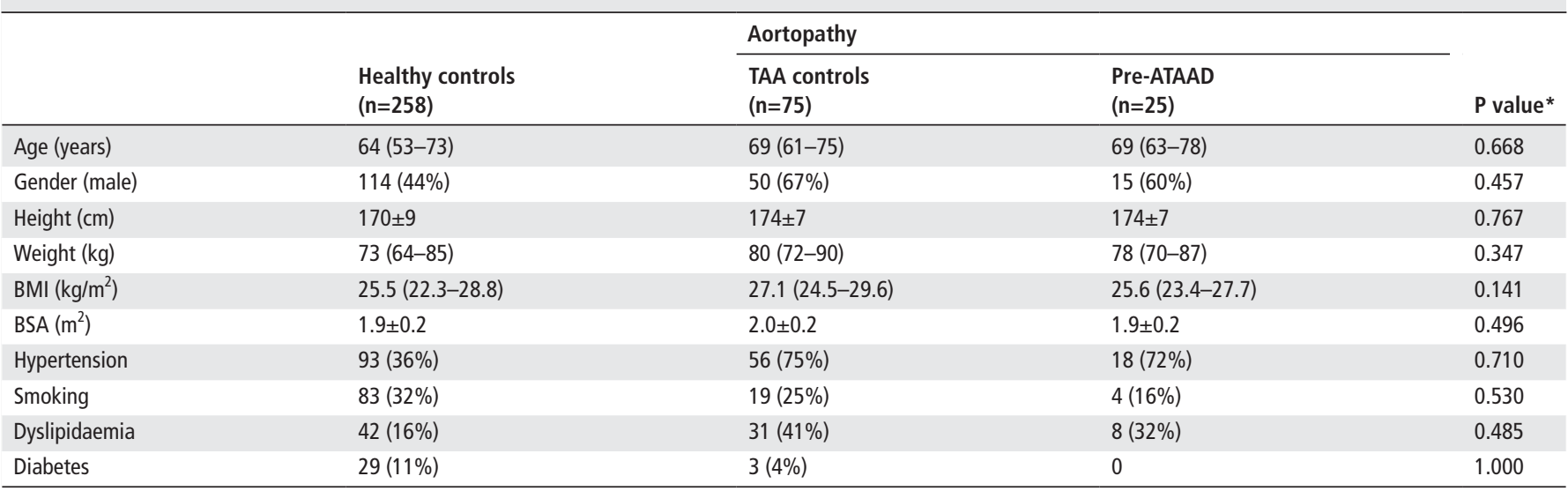

${ }^{*} \mathrm{P}$ values are given for comparison between TAA controls and patients with pre-ATAAD.

ATAAD, acute type A aortic dissection; TAA, thoracic aortic aneurysm. 


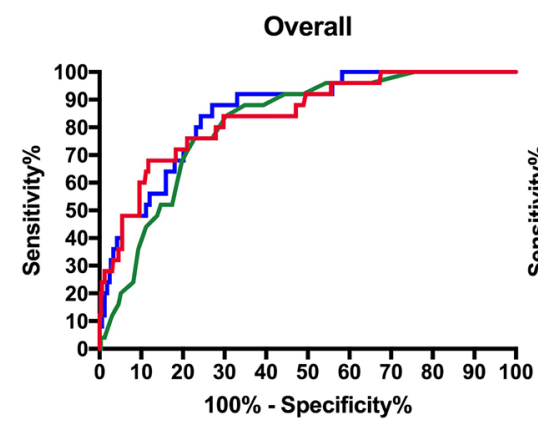

A

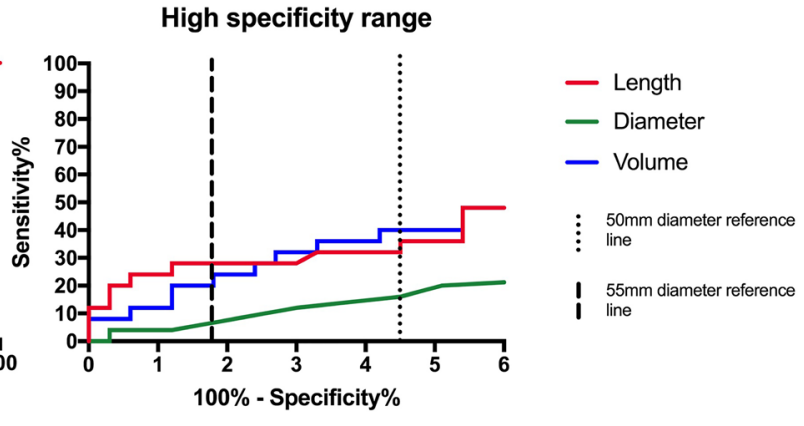

B

Figure 2 Receiver-operating curves for prediction of dissection.

(A) ROC curves comparing the overall ability of different anatomic predictors to identify patients at risk for acute type A aortic dissection. (B) Diameter cut-offs of $50 \mathrm{~mm}$ and $55 \mathrm{~mm}$ yield excellent specificity and preclude unnecessary surgical intervention (dashed lines). At same specificity levels, measurements of aortic length and volume showed significantly higher sensitivities. ROC, receiver-operating curves.

respectively). With ICCs of 0.95 (diameter), 0.94 (length) and 0.99 (volume), interobserver agreement was also excellent. Bland-Altman plots are displayed in figure 3.

\section{DISCUSSION}

Research into the natural risk of TAAs is constrained by multiple methodological issues, one of which is the observation that the dimensions of the aorta change when dissection occurs. ${ }^{15}$ In the current work, we have overcome this limitation by assessing the morphology of the aorta shortly before dissection onset. We found that (i) $96 \%$ of patients with ATAAD fail to meet criteria for prophylactic surgery prior to dissection, (ii) aortic length is significantly larger in patients with pre-ATAAD when compared with TAA controls and (iii) the sensitivities of aortic volume and
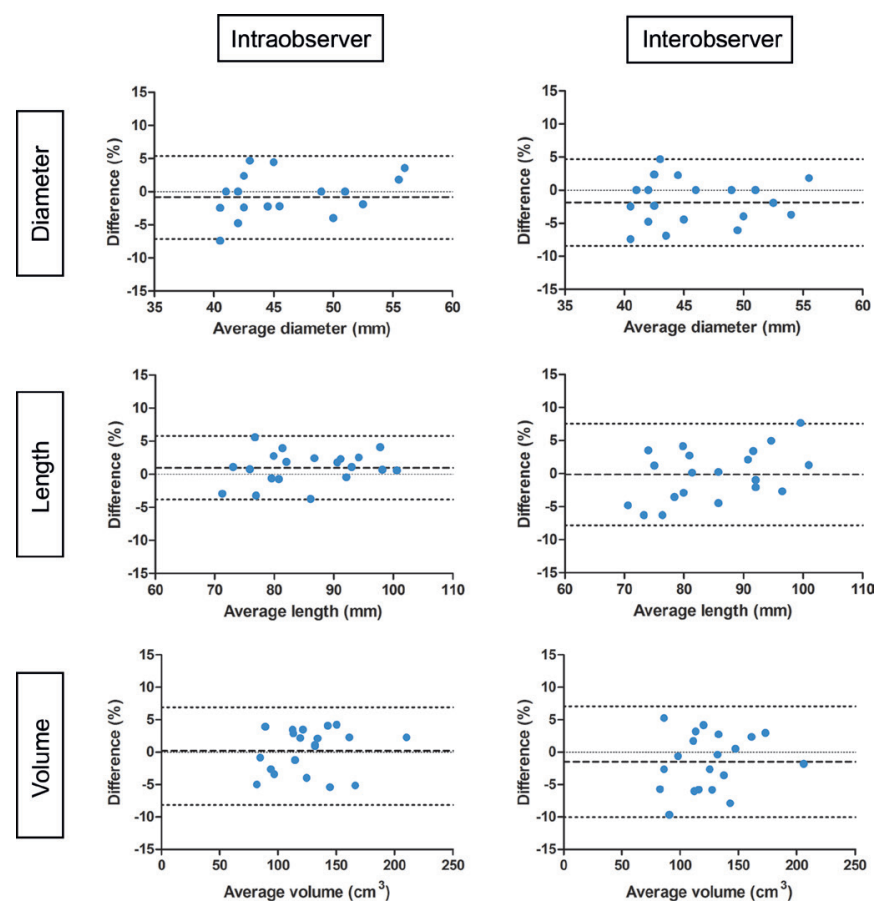

Figure 3 Bland-Altman plots showing intraobserver and interobserver agreement.

Bland-Altman plots showing the normalised bias (dashed line) and 95\% limits of agreement (dotted lines) for measurements of maximal aortic diameter, aortic length and aortic volume. length for prediction of dissection are fivefold and sevenfold higher than the sensitivity of the maximal diameter.

Guidelines for the preventative management of aortic disease depend solely on measurements of maximal cross-sectional dimensions and have long remained unchanged. ${ }^{5}{ }^{6}$ They have largely been based on early work from Davies et al, who longitudinally studied a cohort of patients with TAA and found that the aortic diameter is strongly related to the risk of natural complications. ${ }^{418}$ Even though the cut-off of $55 \mathrm{~mm}$ provides excellent specificity and precludes exposure to unnecessary intervention, it has been well recognised that the current surgical approach is unable to prevent the majority of dissections. ${ }^{8919}$ Our findings confirm estimates by Rylski et al, who modelled postdissection aortas to predissection dimensions and postulated that $>90 \%$ of patients with ATAAD would not have qualified for prophylactic surgery before dissection onset. ${ }^{20}$ As such, they emphasise the need for deciphering additional parameters that can improve patient selection for pre-emptive surgery.

Over the past decade, technological advances have facilitated the acquisition of high-resolution isotropic multislice spiral CT and MRI datasets. Whereas earlier techniques (such as ultrasound and spiral CT) only provided for two-dimensional (2D) images and measurements, modern acquisitions afford a detailed three-dimensional (3D) visualisation of the human arterial system. This evolution has paved the way for more comprehensive radiological measurements, such as those of aortic length and volume shown here. Previous studies investigating vessel length have demonstrated that the healthy aorta gradually elongates with age and that the dissected aorta is notably longer than would have been expected based on age, gender and body surface area (BSA). ${ }^{12-14} 21-23$ The current work adds to this knowledge by revealing that aortic length also serves as more discriminative ATAAD predictor than the maximal transverse diameter. Although the observed sensitivity of $28 \%$ may not seem earth shattering at first glance, it means substantial and readily available improvement in the diseases' detection rate, with no trade-off in terms of specificity. Before the start of the study, we expected aortic volume to evolve into an even better indicator of future adverse events. However, our data have proven otherwise. Whereas the sensitivity of aortic volume was significantly higher than that of the maximal transverse diameter, we found no additional diagnostic value over measurements of aortic length. Yet, in line with previous studies, aortic volume did show excellent reproducibility, which could serve as important argument in favour of its use in clinical TAA follow-up. ${ }^{24}$ Aortic 
length and volume now require longitudinal and prospective validation as the next step towards their implementation into clinical practice guidelines.

It should be noted that current surgical indications are based on imaging data collected in the 1980s and 1990s, a time when measurements were performed mainly on axial images. Since then, and along with the transition to $3 \mathrm{D}$ imaging, the semiautomated centreline method has become routinely available. Multiple previous studies have reported that axial measurements significantly overestimate diameters when compared with centreline methods, especially in more tortuous aortic segments. ${ }^{25} 26$ Based on these observations, it has been argued that current guidelines are not adapted to the use of centreline measurements and that the surgical threshold should be shifted to smaller TAA sizes. ${ }^{27}$ Although we concur that this hypothesis deserves further attention, our data indicate that the diagnostic performance of the maximal diameter-even at lower cut-offs-remains lower than that of aortic volume and length (figure 2B). As expected, lowering the threshold to $50 \mathrm{~mm}$ would increase diameter sensitivity (from 4\% to 17\%). Concurrently, however, the sensitivities of aortic volume (from 20\% to $38 \%$ ) and length (from $28 \%$ to $33 \%)$ would also increase.

\section{Future directions}

Prospective research into the pathophysiology of dissection is challenging due to the diseases' low incidence and the (life) long follow-up duration required for the condition to develop. The design of the current study has afforded an alternative way to evaluate the anatomy of the aorta prior to dissection onset. Our findings could lead to a paradigm shift in the preventative management of aortic disease, enabling the more timely identification of patients at risk. Emerging techniques like fourdimensional (4D) flow MRI and positron emission tomography (PET) permit imaging of the haemodynamic and inflammatory processes that potentially underlie aneurysm progression and could further improve the detection rate of patients who are susceptible to dissection ${ }^{28-30}$

\section{Key messages}

What is already known on this subject?

- The maximal aortic diameter is the principal risk predictor for acute dissection and the sole parameter used for stratification towards pre-emptive aortic replacement. Large registries have demonstrated that more than half of dissection patients present with diameters below the threshold for prophylactic surgery.

\section{What might this study add?}

- Ascending aortic length is significantly increased in dissection patients compared with thoracic aortic aneurysm controls. Measurements of aortic length and volume have superior diagnostic accuracy over diameters for prediction of dissection.

\section{How might this impact on clinical practice?}

- Clinical implementation of aortic length and volume measurements might improve the selection of patients who benefit from preventive aneurysmectomy.

\section{Limitations}

Some limitations of the current study should be addressed. First, a cross-sectional study design was used. Therefore, it is possible that any of the control patients will develop dissection on short terms. Future longitudinal studies are needed to evince a true causal relationship between the various anatomic measurements and the risk of unfavourable outcomes.

Second, the study population is relatively small and not representative of subjects with BAV or connective tissue disease. As the latter are known to suffer dissection at larger aortic diameters, our results are not generalisable to this patient population. ${ }^{9}$ Studies focusing specifically on genetically triggered aneurysms should investigate whether aortic volume and length measurements are of additional value in these patient entities.

\section{CONCLUSION}

The aortic diameter is incapable of predicting the vast majority of dissections. Measurements of aortic volume and length have superior diagnostic accuracy over maximal diameters and could improve the timely identification of patients at risk for ATAAD.

\section{Author affiliations}

${ }^{1}$ Department of Cardiothoracic Surgery, Maastricht University Medical Center, Maastricht, Limburg, The Netherlands

${ }^{2}$ Cardiovascular Research Institute Maastricht (CARIM), Maastricht University, Maastricht, Limburg, The Netherlands

${ }^{3}$ Department of Radiology and Nuclear Medicine, Maastricht University Medical Center, Maastricht, The Netherlands

${ }^{4}$ Department of Cardiology, Maastricht University Medical Center, Maastricht, Limburg, The Netherlands

${ }^{5}$ Department of Cardiovascular Surgery, Heart Centre Freiburg University, Faculty of Medicine, University of Freiburg, Freiburg, Germany

${ }^{6}$ Department of Cardiothoracic Surgery, Catharina Hospital, Eindhoven, The Netherlands

${ }^{7}$ Department of Thoracic and Cardiovascular Surgery, Uniklinik RWTH Aachen, Aachen, Germany

${ }^{8}$ Department of Internal Medicine, Maastricht University Medical Center, Maastricht, Limburg, The Netherlands

${ }^{9}$ Department of Cardiac Surgery, Wroclaw Medical University, Wroclaw, Poland

Contributors $\mathrm{SH}$ and BPA contributed equally to the creation of this manuscript. Study design: SH, BPA, JW, SS and PSN. Data collection: SH, BPA, JRO, BR, MB and KK. Data analysis: SH, BPA, CM. Statistical analysis: SH, HB. Scientific guarantor: JW. Manuscript draft: SH, BPA. Revision, editing and approval of the final manuscript: all authors

Funding This study was supported by Stichting de Weijerhorst, Maastricht, Limburg, the Netherlands

\section{Competing interests None declared.}

Patient and public involvement Patients and/or the public were not involved in the design, or conduct, or reporting, or dissemination plans of this research.

Patient consent for publication Not required.

Provenance and peer review Not commissioned; externally peer reviewed.

Data availability statement Data are available on reasonable request. All participant data and analyses are available on reasonable request.

\section{ORCID iD}

Samuel Heuts http://orcid.org/0000-0002-4409-6206

\section{REFERENCES}

1 Mehta RH, Suzuki T, Hagan PG, et al. Predicting death in patients with acute type A aortic dissection. Circulation 2002;105:200-6.

2 Tanaka Y, Sakata K, Sakurai Y, et al. Prevalence of type A acute aortic dissection in patients with out-of-hospital cardiopulmonary arrest. Am J Cardiol 2016;117:1826-30.

3 Reutersberg B, Salvermoser M, Trenner M, et al. Hospital incidence and In-Hospital mortality of surgically and Interventionally treated aortic dissections: secondary data analysis of the nationwide German Diagnosis-Related group statistics from 2006 to 2014. J Am Heart Assoc 2019;8:e011402. 
4 Davies RR, Goldstein LJ, Coady MA, et al. Yearly rupture or dissection rates for thoracic aortic aneurysms: simple prediction based on size. Ann Thorac Surg 2002;73:17-28.

5 Hiratzka LF, Bakris GL, Beckman JA, et al. 2010 ACCF/AHA/AATS/ACR/ASA/SCA/SCAI/ SIR/STS/SVM guidelines for the diagnosis and management of patients with thoracic aortic disease. J Am Coll Cardiol 2010;55:e27-129.

6 Erbel R, Aboyans V, Boileau C, et al. 2014 ESC guidelines on the diagnosis and treatment of aortic diseases. Eur Heart J 2014;35:2873-926.

7 Kim JB, Spotnitz M, Lindsay ME, et al. Risk of Aortic Dissection in the Moderately Dilated Ascending Aorta. J Am Coll Cardiol 2016;68:1209-19.

8 Pape LA, Tsai TT, Isselbacher EM, et al. Aortic diameter $>0 \mathrm{r}=5.5 \mathrm{~cm}$ is not a good predictor of type A aortic dissection: observations from the International Registry of Acute Aortic Dissection (IRAD). Circulation 2007;116:1120-7.

9 Kim EK, Choi SH, Sung K, et al. Aortic diameter predicts acute type A aortic dissection in patients with Marfan syndrome but not in patients without Marfan syndrome. $J$ Thorac Cardiovasc Surg 2014;147:1505-10.

10 Lindquist Liljeqvist M, Hultgren R, Gasser TC, et al. Volume growth of abdominal aortic aneurysms correlates with baseline volume and increasing finite element analysisderived rupture risk. J Vasc Surg 2016;63:1434-42.

11 Martufi G, Auer M, Roy J, et al. Multidimensional growth measurements of abdominal aortic aneurysms. J Vasc Surg 2013;58:748-55.

12 Adriaans BP, Heuts $S$, Gerretsen $S$, et al. Aortic elongation part l: the normal aortic ageing process. Heart 2018;104:1772-7.

13 Heuts S, Adriaans BP, Gerretsen S, et al. Aortic elongation part II: the risk of acute type A aortic dissection. Heart 2018;104:1778-82.

14 Krüger T, Oikonomou A, Schibilsky D, et al. Aortic elongation and the risk for dissection: the Tübingen aortic Pathoanatomy (taipan) projectt. Eur J Cardiothorac Surg 2017;51:1119-26.

15 Rylski B, Blanke P, Beyersdorf F, et al. How does the ascending aorta geometry change when it dissects? J Am Coll Cardiol 2014;63:1311-9.

16 Stock C, Hielscher T. DTComPair: Comparison of Binary Diagnostic Tests in a Paired Study Design v. 1.0.3. R Statistics, 2014.

17 McNemar Q. Note on the sampling error of the difference between correlated proportions or percentages. Psychometrika 1947;12:153-7.
18 Coady MA, Rizzo JA, Hammond GL, et al. What is the appropriate size criterion for resection of thoracic aortic aneurysms? J Thorac Cardiovasc Surg 1997;113:476-91.

19 Trimarchi S, Jonker FHW, Hutchison S, et al. Descending aortic diameter of $5.5 \mathrm{~cm}$ or greater is not an accurate predictor of acute type B aortic dissection. J Thorac Cardiovasc Surg 2011;142:e101-7.

20 Rylski B, Branchetti E, Bavaria JE, et al. Modeling of predissection aortic size in acute type A dissection: More than $90 \%$ fail to meet the guidelines for elective ascending replacement. J Thorac Cardiovasc Surg 2014;148:944-8.

21 Sugawara J, Hayashi K, Yokoi T, et al. Age-associated elongation of the ascending aorta in adults. J Am Coll Cardiol Img 2008;1:739-48.

22 Krüger T, Forkavets 0 , Veseli K, et al. Ascending aortic elongation and the risk of dissection. Eur J Cardiothorac Surg 2016;50:241-7.

23 Rylski B, Desjardins B, Moser W, et al. Gender-related changes in aortic geometry throughout life. Eur J Cardiothorac Surg 2014;45:805-11.

24 Renapurkar RD, Setser RM, O'Donnell TP, et al. Aortic volume as an indicator of disease progression in patients with untreated infrarenal abdominal aneurysm. Eur J Radiol 2012;81:e87-93.

25 Mendoza DD, Kochar M, Devereux RB, et al. Impact of image analysis methodology on diagnostic and surgical classification of patients with thoracic aortic aneurysms. Ann Thorac Surg 2011;92:904-12.

26 Rudarakanchana N, Bicknell CD, Cheshire NJ, et al. Variation in maximum diameter measurements of descending thoracic aortic aneurysms using unformatted planes versus images corrected to aortic centerline. Eur J Vasc Endovasc Surg 2014;47:19-26.

27 Ziganshin BA, Zafar MA, Elefteriades JA. Descending threshold for ascending aortic aneurysmectomy: Is it time for a "left-shift" in guidelines? J Thorac Cardiovasc Surg 2019;157:37-42.

28 Forsythe RO, Dweck MR, McBride OMB, et al. (18)F-Sodium Fluoride Uptake in Abdominal Aortic Aneurysms: The SoFIA(3) Study. J Am Coll Cardiol 2018;71:513-23.

29 Guzzardi DG, Barker AJ, van Ooij P, et al. Valve-related hemodynamics mediate human bicuspid aortopathy: insights from wall shear stress mapping. J Am Coll Cardiol 2015;66:892-900.

30 Markl M, Frydrychowicz A, Kozerke S, et al. 4D flow MRI. J Magn Reson Imaging 2012;36:1015-36. 